\title{
Myocardial Perfusion Scintigraphy: From Request to Report
}

Bob Bury, Catherine Dickinson, Karen Sheard, and Penelope Thorley

London, U.K.: Informa Healthcare, 2007, 160 pages, $\$ 139.85$

This is a small-sized hardcover book with nice glossy pages. The book consists of 8 chapters focused completely on how to perform gated myocardial perfusion imaging (MPI). It presents practical and concise information about how to start an efficient and organized nuclear cardiology laboratory and perform good-quality gated MPI. It aims to help increase the use of MPI for screening and management of patients with coronary artery disease. The authors clearly and simply outline the details of how to arrange the laboratory; prepare the patient; perform the stress test; and acquire, process, and interpret the nuclear part of the studies. Although brief, the chapters include important, practical information that is sometimes difficult to find in larger textbooks or in the literature. It also touches on every major issue related to MPI. The last chapter emphasizes the role and the importance of MPI, with clear outlines of indications and case scenarios in which MPI was crucial in the patient management plan. The chapter about "stressing the patient" would be the most informative for the noncardiologist, whereas the chapters about scanning and processing the images would be the most informative for beginners in nuclear cardiology. The chapter about image interpretation is useful for all readers. I found the chapter about stressing the patient to be the most informative, with a clear listing of the absolute and relative contraindi- cations, as well as the advantages and disadvantages of each mode of stress testing. I was also impressed by the bold and frank opinion of the authors about many gray areas in the field of MPI. There are many tables throughout the book that can be useful on a routine, daily basis in a nuclear cardiology facility. The abundant illustrations are of good quality, with clear legends, and clarify and support the information presented. Every chapter is followed by a few recent references. The weakness of the book is that it outlines the method of MPI that the authors use in their facility, with no details of other methods of performing MPI. The authors believe that this is the best method of performing MPI in their facility after their long experience in this field of nuclear cardiology.

Overall, the book is easy and interesting to read and can be completed in a short time for the eager reader. This book is a great asset for beginners who would like to start a nuclear cardiology facility and produce good-quality gated MPI studies in a short interval.
Isis W. Gayed

M.D. Anderson Cancer Center

Houston, Texas

COPYRIGHT (๑) 2009 by the Society of Nuclear Medicine, Inc.

DOI: 10.2967/jnumed.108.059675 